\title{
Survey on Assessment of Cognitive States during Learning Activities using Brain Computer Interface based EEG
}

\author{
Pratibha R. Bhise \\ Research Scholar \\ Department of Computer Science \\ and Information Technology, \\ Dr.Babasaheb Ambedkar \\ Marathwada University, \\ Aurangabad, Maharashtra, India
}

\author{
Sonali B. Kulkarni \\ Assistant Professor \\ Department of Computer Science \\ and Information Technology, \\ Dr.Babasaheb Ambedkar \\ Marathwada University, \\ Aurangabad, Maharashtra, India
}

\author{
Talal A. Aldhaheri \\ Research Scholar \\ Department of Computer Science \\ and Information Technology, \\ Dr.Babasaheb Ambedkar \\ Marathwada University, \\ Aurangabad, Maharashtra, India
}

\begin{abstract}
Brain-Computer Interface (BCI) associations the human's neural world and the outer physical world by interpreting individuals' brain signals into commands detectable by computer devices. In BCI cognitive neuroscience is a vital research field. In recent years, increasing studies have employed many technologies to monitor students' cognitive states and attempted to provide adaptive interfaces and contents accordingly to improve learning efficiency of students. As there is a lot of literature on the theory, method and practice of psycho-physiological analysis in BCI context, in this paper we are only covering the part relates to cognitive state estimation with respect to learning activities. Detecting cognitive states is an important step towards adaptive learning because of this reason we move to set the goal of this paper is to review the learning activities and the parameters involved in estimating the cognitive state. According to this study the various authors has done the work on various learning fields such as Mathematics, Engineering, Programming and Medical helps to assess the cognitive states like memory, engagement, mental workload, attention etc. at National and International level.
\end{abstract}

\section{Keywords}

Cognitive States Learning Activities, BCI, Electroencephalography

\section{INTRODUCTION}

Recent research in the field of neuroscience provides a new direction using psycho-physiological techniques to construct more interactive brain computer interfaces (BCI) which can translate human neural responses into control signals for computer-application devices through system's hardware and software. It allows users to communicate via brain initiated control signals without any physical movement in the external world[1],[2].The research area Cognition is one of the important domain in neuroscience field. Cognition is nothing but the working of the human mind or A cognitive state is the mental action of acquiring knowledge through thoughts, experience and senses. There are various processes and functions that contribute to a cognitive state or skills such as attention, working memory, reasoning, Engagement, Perception, problem solving, and so forth. Psychological efforts means the cognitive actions Executed to complete a task. In cognitive psychology the term cognitive load refers to the used amount of working memory resources [3],[4]. The cognitive skill focused attention means all subjects' activities involve active cognitive processes such as problem-solving and critical thinking .whereas the state working memory is a type of short-term memory that allows subjects to store and manipulate temporary information[5].These cognition skills are plays very crucial role in human's life, such as decision making, learning .During the learning cycle whether students are attentive when adapting altogether results learning pointedly impacts their learning outcomes. In traditional faceto-face instruction, instructors generally observe students' expressions to regulate whether they are sufficiently attentive. However, this method is excessively subjective and consumes a significant amount of the teacher's energy[6]. Furthermore, besides face-to-face instruction, students may engage in distance learning over the internet, which further increases the difficulty of determining whether students are attentive. It means the use of technology in education has become increasingly prevalent to aid instructors in making their lectures more vivid and the learning materials more accessible to students[7].However, the use of technology to support realtime monitoring of how well students grasp the materials they learn is still in its infancy. The ability to measure the student's cognitive skills during instruction is essential as they provide valuable feedback to the instructor whether the learning goals have been achieved and to identify the type of intervention needed to improve learning and cognitive states outcomes [8]. So this need is led to the growing interest using psychophysiological Brain Computer Interface (BCI) systems to collect and analyze signals using EEG from the human brain to determine its cognitive state during learning activity [9].The Detecting cognitive states and skills is an important step towards Personalized and Adaptive learning. Owing to the recent advances in neurotechnology and the progress in understanding of brain cognitive functions, improvements of cognitive performance or acceleration of learning process with brain enhancement systems is not out of our reach anymore, on the contrary, it is a tangible target of contemporary research.As there is a lot of literature on the theory, method and practice of psycho-physiological analysis in BCI context, in this paper we are only covering the part relates to cognitive state estimation with respect to learning using Brain Computer Interface based EEG.

\section{RELATED WORK}

A cognitive state is the mental action of acquiring knowledge through thoughts, experience and Senses. There are many processes and functions that contribute to a cognitive state such as attention, memory, reasoning, problem solving, and so 
forth. For assessment of cognitive states learning module plays very important role. In below section states the research work done by various authors at National and International level in this respective area.

\subsection{Estimation of Cognitive states during learning activities using EEG}

Chris Berka, Daniel J. Levendowski, Michelle N. Lumicao et.al.,[2007] investigated the investigation of observing Electroencephalography(EEG) signs of engagement and workload which was gained and measured during execution of cognitive test.

Thanh A Nguyen , Yong Zeng, [2010] utilized EEG to record designer's cerebrum electrical signs when s/he was chipping away at a design task. Six channels of the EEG signals were recorded, including Fp1, Fp2, Fz, Cz, Pz, Oz, in light of which the power spectral density for every EEG band (delta, theta, alpha and beta) was determined. The outcomes indicated that, for the given deign issue, the subject burned through more energy in visual deduction during the solution generation than that in arrangement assessment.

Yongchang Li , Xiaowei Li [2011] This investigation portray a framework dependent on an Electroencephalogram which gauges the three consideration levels and grouped by a KNN classifier dependent on the Self-Assessment Manikin model with $57.05 \%$ exactness

Adreas Fink , Daniela Schwab et.al .,[2011] researched two methodologies, for example, regardless of whether imaginative insight can be improved by methods for psychological and emotional incitement exercises and whether these mediations are related with changes of EEG alpha action.

Dan Szafir , Bilge Mutlu ,[2012]they draw on procedures from brain computer interfaces (BCI) and information from instructive brain science to plan versatile specialists that screen understudy consideration continuously utilizing estimations from electroencephalography (EEG) and recover reducing consideration levels utilizing verbal and nonverbal signals.

Ning - Han Liu , Cheng - Yu Chiang and Hsuan - Chin Chu [2013] perceived the understudies were mindful and oblivious during learning movement utilizing portable sensors. A support vector machine (SVM) classifier was utilized to compute and dissect these highlights to distinguish the mix of highlights that best shows whether understudies are mindful. In view of the analysis results, the technique proposed in this investigation gives a characterization precision of up to $76.82 \%$. The investigation results can be utilized as a source of perspective for learning framework plans later on.

Kavitha P Thomas , A .P .Vinod,[2013] the authors explore the effect of a neurofeedback put together BCI game with respect to the improvement of attention and intellectual abilities of healthy subjects and the trial aftereffects of this examination show that the proposed neurofeedback preparing model starts the player to extend his entropy scores, upgrade attention level and achieve higher focuses in the game.

Nanda Nandagopal, Vijayalakshmi R, et.al ,[2013] In this examination the authors presents a diagram of the utilization of such strategies to EEG information ,bringing together an assortment of methods containing complex network investigation, coherence ,common data ,inexact entropy ,computer visualization, signal handling and multivariate procedures, for example, the one-way analysis of variance
(ANOVA). This examination exhibits that the incorporation of these strategies empowers a profundity of comprehension of complex cerebrum elements that is unimaginable by different techniques just as permitting the ID of contrasts in framework intricacy that are accepted to underscore typical human insight.

Hyunjeong Lee ,[2013] ,This investigation inspected a solid and legitimate technique for surveying psychological burden during learning through looking at different kinds of intellectual burden estimations: Electroencephalography (EEG), self-announcing, and learning result.

Geeta U .Navalyal , Rahul D .Gavas , [2014], point of this investigation is to detail a technique with the assistance of Brain Computer Interface game to help the coaches in noticing and assessing the attention levels of the students ,at normal intervals during the preparation time frame. For this examination reason the gaming climate is planned utilizing Open Source Graphics Library (OpenGL) package and the game control is through the cerebrum waves of player's utilizing the Brain Computer Interface (BCI) innovation.

Y .Liu , J.M .Ritchie ,et.al ,2014] proposes and examines a system to decide the passionate perspectives credited to a bunch of CAD configuration undertakings by breaking down the CAD administrators' psycho-physiological signs. A fuzzy logic model was set up to plan the psycho-physiological signs to a set of key feelings, to be specific disappointment, fulfillment, commitment and challenge and the outcomes analyzed. Understanding of every participant's feelings were effectively completed with substantial connections showed between the related architects' CAD exercises and their detailed emotional states.

Niannian Wang, Li Zhang, et.al ,[2015],they investigating the cognitive functions of the brain by making an network model to comprehend the working system of the brain has become an exceptionally famous exploration in point in the neuroscience field and in this examination electroencephalography (EEG) was utilized to gather information from subjects given four diverse numerical psychological errands: present numbers clockwise and counter-clockwise, and letters clockwise and counter-clockwise to fabricate a complex brain function network (BFN).

Poulami Ghosh ,Ankita Mazumder et.al [2015] the target of this investigation was to assess the cognitive state of brain. They concentrated in on memory and attention state. For this classification purpose they used Support Vector Machine and they acquired $79 \%$ classification precision.

Necmettin Firat Ozkan , Emin Kahya [2015] An investigation was directed with 30 participants. Every member finished two assignments through a BCI and filled NASA-TLX structures. The outcomes were examined utilizing combined t-tests to see whether BCI undertakings are altogether extraordinary regarding making cognitive load. The consequences of this investigation demonstrated that NASA-TLX scores of the BCI errands were essentially extraordinary and these frameworks can be considered for assessing cognitive examinations.

Patricia Soto-Icaza ,Francisco Aboitiz et.al[2015], Aim of this investigation was initially examine the improvement of social abilities in youngsters ,to explain the behavioral neural component identified with the obtaining of social aptitudes during earliest stages and their appearance as expected Second they quickly depict how formative illnesses like Autism Spectrum Disorders(ASD) can educate about the 
neurobiological systems of social abilities. At long last they draw general system for the elaboration of cognitive models to encourage the perception of human social growth.

Xiaowei Li ,Martyn Ratcliffe et .al.,[2015]describe Real-time EEG - based BCI framework which estimates attention level. In this investigation they contrast their methodology and conventional methodologies, three consideration levels were ordered by a KNN classifier dependent on the SelfAssessment Manikin (SAM) model.

Fumihiko Taya ,Yu Sun ,et.al[2015] recommended that the learning cycle during the cognitive training can be encouraged by an assistive framework checking cognitive workloads with electroencephalography (EEG) biomarkers, and the brain connectome approach can give extra important biomarkers to encouraging leaners' learning measures.

Pouya Bashivan , Irina Rish ,et.al ,[2016], The authors observed reactions to two distinct sorts of input: instructional ('logical') versus recreational ('emotional') recordings, utilizing a scope of AI techniques. They attempted SVMs, sparse logistic regression, and Deep Belief Networks, to segregate between the perspectives prompted by various sorts of video input that can be generally marked as 'logical' versus 'emotional'. Their outcomes showed a major capability of wearable EEG gadgets in separating cognitive states between circumstances with major logical however unobtrusive clear contrasts.

Amit Desai [2017] gained the signs got from the brain are handled to quantitatively study and look at the brain exercises of coders while programming in two distinctive programming languages. In this exploration, they have picked the organized programming language $\mathrm{C}$ and the scripting language Python for examination.

Explored by Xi Liu , Pang - Ning Tan, et.al [2017],the possibility of utilizing EEG demos created from an off-theshelf, wearable gadget to consequently characterize the cognitive conditions of students as they were approached to play out a progression of perusing and question noting assignments. They demonstrated that the EEG information can adequately foresee whether an understudy is mindful or occupied just as the student's understanding rate, which is a significant proportion of understanding familiarity.

Raheel Zafar ,Sarat C.Dass et.al,[2017], In this examination, a novel calculation is proposed to interpret brain movement related with various sorts of pictures. In this hybrid algorithm , convolutional neural network is changed for the extraction of highlights, a t-test was utilized for the choice of critical highlights and probability proportion based score combination was utilized for the expectation of brain action. The strategies utilized in this examination were given the exactness like $65.7 \%$ and $79.9 \%$ separately.

Winnie K .Y.So , Savio W.H.Wong , et.al ,[2017] They researched the achievability of utilizing short term frontal EEG as a way to assess the dynamic changes of mental outstanding task at hand. In this examination subjects were performing four cognitive and motor tasks, including including arithmetic operation, finger tapping, mental rotation and lexical decision task. The degree of mental workload could be ordered from EEG highlights with $65 \% \pm 75 \%$ precision across subjects utilizing a SVM model.

Richard W. Montgomery , Leslie D. Montgomery [2018], This paper depicts how ERP energy density examination and marginal cost-benefit analysis investigation were joined to understand the consequences of an examination of cognitive execution .They shows the cognitive presentation of each subject through four stages, for example, learning ,weariness, inspiration and weakness.

Author J.J.J.Davis , R.Kozma ,[2018] presented a cognitive modalities, for example, open eyes with visual stimuli, open eyes, close eyes, math problem solving and meditation. They demonstrated beginning proof that outer tangible information encourage neural movement in a scope of frequency bands, while self-initiated applied our film making procedure to subjectively investigate brain elements utilizing the art of encephalography in a novel way. In this current study we check the technology employing a 256 electrodes dense-array EEG established by EGI. The applied temporal and spatial Power Spectrum Density analysis is ready to categorize between these modalities.

Asma Ben Khedher, Imene Jraidi et.al,[2018 ] keen on breaking down the connection between students' visual behaviour and their exhibition while resolving clinical cases. Firstly they investigate how the students visually investigate the learning environment across various area of interest Secondly observe whether static and dynamic eye tracking measurements can affect student' thinking execution

In 2019 the authors saw a higher commitment list during the treatment identifictaion stage since it produced more mental exertion additionally statistically major impacts were found between metal engagement and students exhibition .This examination will valuable for comprehension of the student learning experience.

Muhammad Zeeshan Baig ,Manolya Kavakli [2019] They introduced a relative investigation of novice/expert data stream designs. Normalized Transfer Entropy (NTE) and Electroencephalogram (EEG)was used to examine the distinctions. The test was isolated into three cognitive states i.e., rest, drawing, and manipulation. They applied characterization calculations on NTE frameworks and diagram hypothesis measures to see the adequacy of NTE and accomplished over $90 \%$ exactness with a straightforward Knearest neighbors $(\mathrm{k}-\mathrm{NN})$ to categorize novice and expert clients.

The authors Antoine Gaume ,Gerard Dreyfus et.al[2019] present a cognitive brain computer interface dependent on a persistent execution task for the checking of varieties of visual supported consideration, for example self-directed maintenance of cognitive concentration in non-stimulating conditions while potentially disregarding distractors and keeping away from mind wandering. Generalization execution assess for pairwise arrangement of assignment trouble utilizing these highlights reached $75 \%$ for $5 \mathrm{~s}$ ages, and $85 \%$ for $30 \mathrm{~s}$ ages.

These Aurelien Appriou, Andrzej Cichocki et.al authors in this paper investigates such machine learning algorithms, proposes new variations of them, and benchmarks them with traditional strategies to appraise both mental remaining burden and full of feeling states (Valence/Arousal) from EEG signals. They study these methodologies with both subjectexplicit and subject-autonomous adjustment, to go towards alignment free frameworks. Their outcomes proposed that a CNN got the most noteworthy mean exactness, albeit not fundamentally in this way, in the two conditions for the psychological remaining task at hand study, trailed by RGCs. Nonetheless, this equivalent $\mathrm{CNN}$ failed to meet expectations 
in the two conditions for the feeling informational index, an informational collection with little preparing information. Despite what might be expected, RGCs demonstrated to have the most elevated mean precision with the Filter Bank Tangent Space classifier (FBTSC) they presented in this paper. Their outcomes subsequently added to improve the dependability of intellectual and emotional states characterization from EEG. They additionally give rules about when to utilize which AI calculation.

\section{CONCLUSION}

Recent years, there has been increasing interest in utilizing EEG signals to determine the cognitive state of students as they engaged in various learning activities. Detecting cognitive skill is a crucial step towards adaptive learning. This review contains the assessment of cognitive states during learning activities. In particular, the researchers has done mostly work on cognitive states such as attention, workload, inattention, memory and engagement in the field of medical ,engineering ,programing and mathematics with the help of BCI based EEG. As we know the ability to recognize and measure student's mental state during learning process is an important part of a successful knowledge acquisition since it impacts their cognitive performance. This study useful to obtaining an accurate view of learner's mental state at the time of learning activities using BCI based EEG which helps to students for enhancing their cognitive skills as well as learning.

\section{ACKNOWLEDGMENTS}

Authors would like to acknowledge to Department of Computer Science \& IT, Dr. Babasaheb Ambedkar Marathwada University, Aurangabad, Maharashtra, India. And financial assistance under Rajiv Gandhi National Fellowship for this work.

\section{REFERENCES}

[1] Lan T, Adami , A ,et.al," Estimating Cognitive State Using EEG Signals", In Proceedings of the 2005 13th European Signal Processing Conference, Antalya, Turkey, 4-8 , pp. 1 4,September 2005

[2] DraganskiB.,Gaser,C.,Kempermann,G.,Kuhn,H.G.,Wink ler,J.,Büchel,C., et.al, “ Temporal and spatial dynamics of brain structure changes during extensive learning.", $J$. Neurosci. 26, 6314-6317.doi:10.1523/jneurosci.462805.2006

[3] Dimitriadis,S.I.,Sun,Y.,Kwok,K.,Laskaris,N.A.,Thakor,N ., and Bezerianos,A ," Cognitive work load assessment based on the tensorial treatment of EEG estimates of cross-frequency phase interactions", Ann. Biomed.Eng.

[4] Lotte, F. et al.: A Review of Classification Algorithms for EEG-based Brain-Computer Interfaces. J. Neural Eng. 4, 1-24 (2007).

[5] Chris Berka, Daniel J. Levendowski, Michelle N. Lumicao et.al.," EEG Correlates of Task Engagement and Mental Workload in Vigilance, Learning, and Memory Tasks “,Aviation ,Space and Environmental Medicine,Vol.78,pp.231-244,2007.

[6] Thanh An Nguyen, Yong Zeng, "Proceedings of the ASME 2010 International Design Engineering Technical Conferences \& Computers and Information in Engineering Conference, 2010.

[7] Yongchang Li , Xiaowei Li, et .al,"A Real-time EEGbased BCI System for Attention Recognition in
Ubiquitous Environment “,Researchgate,2011

[8] Andreas Fink , Daniela Schwab et.al , Sensitivity of EEG Upper Alpha Activity To Cognitive and Affective Creativity Interventions ",International Journal of Psychophysiology (ELSEVIER),pp.233-239,2011.

[9] Dan Szafir , Bilge Mutlu ,'Pay Attention ! Designing Adaptive Agents That Monitor and Improve User Engagement “, Researchgate ,2012.

[10] Ning-Han Liu , Cheng -Yu Chiang et.al ,’Recognizing the Degree of Human Attention Using EEG Signals from Mobile Sensors ”, Sensors , ISSN .1424-8220 ,pp.1027310286,2013 .

[11] Kavitha P.Thomas , A .P .Vinod ," Enhancement of Attention and Cognitive Skills Using EEG Based Neurofeedback Game ", $6^{\text {th }}$ Annual International IEEE EMBS Conference on Neural Engineering San Diego,California,2013

[12] Nanda Nandagopal, Vijayalakshmi R , et.al,"Computational Techniques for Characterizing Cognition Using EEG Data-New Approaches ", $17^{\text {th }}$ International Conference in Knowledge Based and Intelligent Information and Engineering Systems-KES 2013(ELSEVIER),pp.699-708,2013

[13] Hyunjeong Lee ,"Measuring Cognitive Load with Electroencephalography and Self -Report :Focus on the effect of English - Medium Learning for Korean Students ",An International Journal of Experimental Education Psychology ,ISSN.0144-3410,Vol.34,pp-838-848,2013

[14] Bashivan, P. et al.," Neural correlates of visual working memory load through Unsuvised spatial filtering of EEG", In: Proceedings of 3rd workshop on Machine Learning and interpretation in neuroimaging. (2013).

[15] Bashivan, P. et al.,"Spectro temporal dynamics of the EEG during working memory encoding and maintenance predicts individual behavioral capacity", Eur. J. Neurosci. 40, 12, 3774-3784 (2014).

[16] Geeta U .Navalyal , Rahul D .Gavas ,” A Dynamic Attention Assessment and Enhancement Tool Using Computer Graphics",Human-Centric Computing and Information Sciences ,2014

[17] Y.Liu , J.M. Ritchie ,et.al ,’A Fuzzy Psychophysiological Approach to Enable the Understanding of an Engineer's Affect Status During CAD Activities", Computer Aided Design,pp.19-38,2014

[18] Ke .Y ,Qi .H ,et.al ,” An EEG-based mental workload estimator trained on working memory task can work well under simulated multi-attribute task. Front. Hum. Neuroscience, 8, 703.2014

[19] Poulami Ghosh ,Ankita Mazumder et.al ," An EEG Study on Working Memory and Cognition",Proceedings of the $2^{\text {nd }}$ International Conference on Perception and Machine Intelligence ,pp.21-26,2015.

[20] Niannian Wang, Li Zhang, et.al ,"EEG-based Research on Brain Functional Networks in Cognition ", BioMedical Materials and Engineering,pp.1107-1114,2015

[21] Necmettin Firat Ozkan , Emin Kahya ," An Experiment in Use of Brain Computer Interfaces for Cognitive Researchers “, International Journal of Intelligence Science ,pp.80-88,2015 
[22] Patricia Soto-Icaza „Francisco Aboitiz et.al ," Development of Social Skills in Children : Neural and Behavioral Evidence for the elaboration of Cognitive Models “, Frontiers in Neuroscience ,Volume .9 2015.

[23] Xiaowei Li ,Martyn Ratcliffe et.al ," A Real -time EEGbased BCI System for Attention Recognition in Ubiquitous Environment”,Researchgate,pp.33-39,2015

[24] Fumihiko Taya , Yu Sun , et.al ,'Brain Enhancement Through Cognitive Training : A New Insight From Brain Connectome “, Frontiers in Systems Neuroscience ,Vol.9, pp.1-19,2015

[25] Pouya Bashivan , Irina Rish ,et.al ,'Mental State Recognition via Wearable EEG",2016

[26] Raheel Zafar ,Sarat C.Dass et.al ," Electroencephalogram-based Decoding Cognitive States Using Convolutional Neural Network and Likelihood Ratio Based Score Fusion “,PLOS ONE ,pp.1-23, 2017

[27] EEG-Based Evaluation of Cognitive and Emotional Arousal When Coding in Different Programming Languages by Amit Rajendra Desai,2017

[28] Xi Liu , Pang -Ning Tan, et.al ," Automated Classification of EEG Signals For Predicting Student's Cognitive State During Learning",pp.1-8,2017 .

[29] Winnie K .Y .So , Savio W .H .Wong, et.al ," An Evaluation of Mental Workload with Frontal EEG “,PLUS ONE ,pp.1-17,2017

[30] Mohammadpour, M.; Mozaffari, S. Classification of EEG-Based Attention for Brain Computer Interface.In Proceedings of the 2017 3rd Iranian Conference on Intelligent Systems and Signal Processing, Shahrood, Iran, 20-21 , pp. 34-37, December 2017

[31] Richard W. Montgomery, Leslie D. Montgomery, ,EEG Monitoring of Cognitive Performance ",Physical Medicine and Rehabilitation Research ,ISSN.23983353,Vol.3(4),pp-1-5,2018

[32] J .J. J.Davis ， R.Kozma ,"Visualization of Human
Cognitive States Monitored by High Density EEG Arrays ",INNS Conference on Big Data and Deep Learning (ELSEVIER),ISSN.1877-0509,pp.219-231,2018.

[33] Asma Ben Khedher , Imene Jraidi et.al,"Static and Dynamic Eye Movement Metrics for Student's Performance Assessment",Smart Learning Environment(Springer),pp.1-12,2018

[34] Zainab Mohamed , Mohamed El Halaby et.al ," Characterizing Focus Attention and Working Memory Using EEG “,Sensors,pp.1-21,2018

[35] Muhammad Zeeshan Baig, Manolya Kavakli ,"A Survey on Psycho-Physiological Analysis \& Measurement Methods in Multimodal Systems “,Multimodal Technologies and Interact (MDPI),2019

[36] Muhammad Zeeshan Baig ,Manolya Kavakli ,"Connectivity Analysis Using Functional Brain Networks to Evaluate Cognitive Activity During 3D Modelling “,Brain Sciences ,pp.1-20,2019

[37] Antoine Gaume ,Gerard Dreyfus et.al,"A Cognitive Brain Computer Interface Monitoring Sustained Attentional Variations During a Continuous Task",Cognitive Neurodynamics,Vol.13.pp.257269,2019

[38] Aurelien Appriou , Andrzej Cichocki ,et.al ," Modern Machine Learning Algorithms to Classify Cognitive and Affective States from Electroencephalography Signals", IEEE, Feb 2020.

[39] Pratibha R .Bhise ,Sonali B.Kulkarni, Talal A.Aldhaheri ,"Brain Computer Interface Based EE for Emotion Recognition System :A Systematic Review ", $2^{\text {nd }}$ International Conference on Innovative Mechanisms for Industry Applications (IEEE),March 2020.

[40] Talal A.Aldhaheri, Sonali B.Kulkarni, Pratibha R .Bhise ," Brain Computer Interface and Neuro Linguistics : A Short Review ", $2^{\text {nd }}$ International Conference on Sustainable Communication Networks and Application (Springer),August 2020. 